\title{
EFEITO DA SUPLEMENTAÇÃO LIPÍDICA PARA MATRIZES SUÍNAS NO PERÍODO PÓS PARTO SOBRE SEU INTERVALO DESMAME-ESTRO E, VARIAÇÃO DE PESO E ESCORE CORPORAL
}

\author{
(Effect of lipid supplementation for sows in the postpartum period on its wean-to-estrus interval, weight \\ variation and body condition score)
}

Marley Conceição dos Santos, Kariny Fonseca da Silva, Letícia Dzierva, Isabella de Camargo Dias, Francielle de Oliveira Marx, Vitor Augusto Bernardini Zavelinski, Alex Maiorka

Universidade Federal do Paraná, Curitiba, Paraná, Brasil.

*Correspondência: marleyconceicaos@gmail.com

RESUMO: O período pós parto é de grande desafio nutricional para matrizes suínas, por conta disso, este trabalho buscou avaliar alternativas viáveis para diminuir a perda de peso e escore de condição corporal das porcas durante a lactação, bem como seu intervalo desmame-estro subsequente, testando a suplementação de óleo de soja, em cinco níveis, para 300 matrizes nos três primeiros dias pós parto. Os tratamentos foram: CO - Controle (apenas o óleo já presente na ração $-604,5 \mathrm{~g}), T 750-750 \mathrm{~g}, \mathrm{~T} 1000-1000 \mathrm{~g}, \mathrm{~T} 1250$ - 1250g e T1500 - 1500g, com 60 animais por tratamento. Não foi observada diferença significativa para variação de peso e escore corporal das matrizes entre os tratamentos durante o período experimental, assim como sobre 0 IDE $(P>0,05)$. Esse resultado provavelmente se deve ao que foi relatado por Zhang et al. (2018), que mostram que nos três primeiros dias de vida as porcas ainda não estão consumindo a quantidade de ração suficiente para suprir sua demanda nutricional, portanto mesmo a suplementação lipídica pode ser insuficiente para alcançar os níveis demandados. Considerando as condições experimentais deste estudo, a suplementação de óleo de soja para as matrizes nos três primeiros dias pós parto não tem efeito sobre a sua perda de peso e condição corporal na fase de maternidade, e não influencia seu intervalo desmame-estro subsequente.

Palavras-Chave: nutrição de suínos; óleo de soja; lactação.

Nota: Este projeto foi aprovado pela Comissão de Ética no Uso de Animais do Setor de Ciências Agrárias da Universidade Federal do Paraná, Curitiba, PR, Brasil, sob o protocolo 087/2018.

Referência: ZHANG, S.; CHEN, F.; ZHANG, Y.; LV, Y.; HENG, J.; MIN, T.; LI, L.; GUAN, W. Recent progress of porcine milk components and mammary gland function. Journal of Animal Science and Biotechnology, China. n. 9, v. 77, 13p, 2018. 